Optical Society of America. V. S. Sudarshanam and S. B. Desu, "Fiber-optic polarization and phase modulator utilizing transparent piezofilm with indium tin oxide electrodes," Appl. Opt. 34, 1177-1189 (1995). doi: 10.1364/AO.34.001177

\title{
Fiber-optic polarization and phase modulator utilizing transparent piezofilm with indium tin oxide electrodes
}

\author{
V. S. Sudarshanam and S. B. Desu
}

\begin{abstract}
A highly efficient optical polarization and phase modulator formed by the placement of a thin transparent piezofilm with indium tin oxide electrodes directly in the path of the output from an optical fiber is presented. Various configurations that differ in the clamping conditions, utilization of epoxy, and optical arrangement are presented. For a film thickness of $63.9 \mu \mathrm{m}$, a linear phase-shifting coefficient of 0.131 $\mathrm{rad} /$ voltage peak $(\mathrm{Vp})$ at $2 \mathrm{kHz}$ and of $0.508 \mathrm{rad} / \mathrm{Vp}$ at $7.4 \mathrm{kHz}$ is demonstrated. An intrinsic birefringence of 0.0328 between the directions along the stretch and its perpendicular in the plane of the film has been measured. The polarization modulation coefficient was determined to be $0.323 \mathrm{rad} / \mathrm{Vp}$ at $8.423 \mathrm{kHz}$, corresponding to a half-wave voltage of $8.353 \mathrm{Vp}$. Applications of the device involving concurrent spatiotemporal polarization and phase modulation are indicated.

Key words: Phase modulator, polarization modulator, fiber-optic devices, transparent piezofilms, optical interferometry, signal processing, transmission ellipsometry.
\end{abstract}

\section{Introduction}

Optical phase and polarization modulators ${ }^{1-4}$ form an integral part of fiber-optic interferometric sensor systems for phase calibration and for the implementation or testing of several detection schemes that extract the signal of interest from the ambient optical, electronic, and mechanical noise. Such modulators can be of the bulk-optic kind, and they have been utilized extensively in a variety of holographic, bulkoptic, and fiber-optic interferometers. In comparison with the emphasis in holographic and bulk-optic interferometers, the emphasis in fiber-optic sensor systems has been on compactness, low insertion losses, low drive voltages, and flexibility of design. Thus, in preference to the use of bulk-optic components, in-line, all-fiber phase and polarization modulators have been devised over the last decade wherein the light remains guided within the fiber in the interaction region of the modulator. For example, such an interaction is commonly achieved by bonding the fiber onto a piezoelectric lead zirconate titanate

The authors are with the Department of Materials Science and Engineering, Virginia Polytechnic Institute and State University, Blacksburg, Virginia 24061-0237.

Received 6 December 1993; revised manuscript received 11 May 1994.

0003-6935/95/071177-13\$06.00/0.

(c) 1995 Optical Society of America. cylinder or a thin strip of polyvinylidene fluoride (PVDF) film. ${ }^{1-4}$ Modulators in which bonding is brought about by the use of an adhesive or epoxy as a medium for transfer of strain from the strictive element to the fiber have been shown ${ }^{3,4}$ to exhibit a large nonlinearity of response and reduced strain transfer efficiency resulting in a low value for the phase-shifting coefficient (PSC) and the dynamic range. The reduction in the strain transfer efficiency occurs because of the different elastic parameters of the materials forming the fiber, the piezofilm, and the epoxy. These limitations become pronounced when longer fiber lengths are used to increase the integral phase and polarization modulation, because longer fiber lengths imply a larger volume of epoxy used. Further, multiple passes of the fiber over the interaction region become necessary if a long fiber is used, thus leading to the requirement that the device be designed to be compact. This, however, is not possible in configurations that utilize a single flat strip of the strictive element. ${ }^{4}$

Whereas in such modulators time-dependent modulation alone has been considered and has been necessary, several applications exist that require concurrent spatial modulation ${ }^{5}$ along with temporal modulation. This paper presents a fiber-optic phase and polarization modulator utilizing a thin piezoelectric PVDF film with optically transparent electrodes made of indium tin oxide (ITO). The piezofilm is 
placed directly in the path of the fiber output as in a bulk-optic modulator configuration, with the difference that the interaction length (the optical thickness of the film) is far smaller than that of the singlecrystal modulators. The device performance characterization and demonstration of reliability for the several different configurations reported herein indicate the utility of directly depositing the film and the electrodes on the end face of a fiber, thus combining the advantages of the fiber geometry and the longitudinal modulator configuration. Compared with several meters of fiber interaction length used in existing fiber modulator configurations, a single thin film of $\sim 64 \mu \mathrm{m}$ is shown to provide the same order of magnitude of the PSC. The modulator was tested with a combined Mach-Zehnder-Fabry-Perot interferometric configuration for measuring the phase shift, and a typical transmission ellipsometric arrangement for the measurement of polarization modulation. In contrast to in-line all-fiber polarization modulators, the utility of the PVDF film for polarization modulation has several advantages, such as (a) easy identification of fast and slow axes that are permanently fixed and do not change with time and external disturbances as in the optical fiber, (b) a high value of intrinsic birefringence, and (c) a large modulation depth for a given applied voltage with a shorter interaction length.

The experimental arrangement and signal detection schemes are described in Section 2, followed by the experimental results for both phase and polarization modulation in Section 3. The experimental measurement of the piezofilm birefringence and thickness as well as the origin of polarization modulation in an electrically driven PVDF film are also discussed in Section 3. From the experimental results obtained, the utility of the device for applications in optical systems of the holographic, bulk-optic, and fiber-optic kinds is indicated in Section 4, followed by the conclusion in Section 5.

\section{Experimental Arrangement and Signal Detection}

We measured the transmission spectrum of the PVDF piezofilm ${ }^{6}$ along with the ITO electrodes by using an ultraviolet-visible-near-infrared scanning spectrophotometer (Shimadzu UV-3101PC); the spectrum is shown in Fig. 1. As we see from Fig. 1, for the particular sample of the piezofilm used, the transmittance at $633 \mathrm{~nm}$ was $71.77 \%$, whereas that at the IR wavelengths of 1300 and $1550 \mathrm{~nm}$ were $89.81 \%$ and $88.71 \%$, respectively. However, for convenience, the source utilized was a He-Ne laser operating at 633 nm. The plot in Fig. 1 shows a distinct fringe pattern of appreciable modulation depth, especially in the regions of higher wavelengths. The utility of this fringe pattern for the estimation of the thickness of the film is discussed in Subsection 3.B.

\section{A. Phase-Shift Measurement through Interferometry}

A hybrid fiber-optic Mach-Zehnder interferometer was formed from a fused-fiber directional coupler and

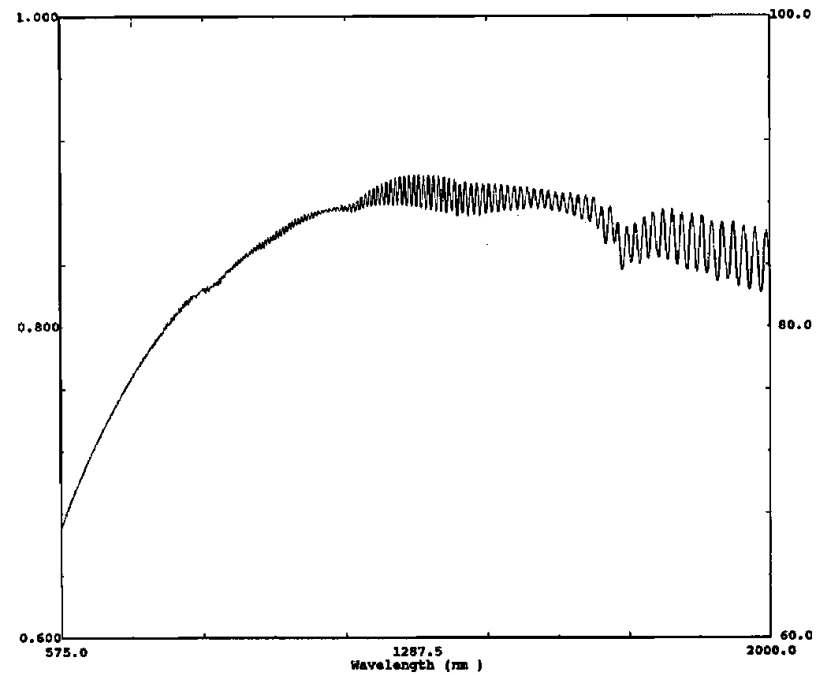

Fig. 1. Transmission spectrum of the PVDF film with the ITO electrodes as measured by the ultraviolet-visible-near-infrared scanning spectrophotometer. The vertical axis is marked on the left-hand side in dimensionless units of transmittance, whereas the right-hand side is in terms of corresponding percentage of transmission.

a beam splitter, as shown in Fig. 2. The output of the Mach-Zehnder interferometer was accessed by detector D1. The modulator consisting of the piezofilm and the mounting arrangement is hereafter referred to as the IPM, standing for ITO-coated PVDF (phase polarization) modulator. The IPM was placed at the end of the signal fiber as shown in Fig. 2. The reflections from the electrode surfaces of the piezofilm would reach detector D2 through the signal fiber and the directional coupler. Thus, an extrinsic fiberoptic Fabry-Perot interferometer ${ }^{7}$ is formed at the piezofilm concurrently with the Mach-Zehnder interferometer. The reflection from the end face of the reference fiber would also reach detector D2, and on recombination with the reflections from the IPM surfaces, form the output of a Michelson interferometer that is sensitive to path differences between the signal and the reference fibers. To eliminate this effect, we terminated the reference fiber in indexmatching liquid. ${ }^{8}$ The output from the Fabry-Perot interferometer (FPI) at D2 would be more robust against random phase drifts than that from the Mach-Zehnder interferometer (MZI) seen at D1.

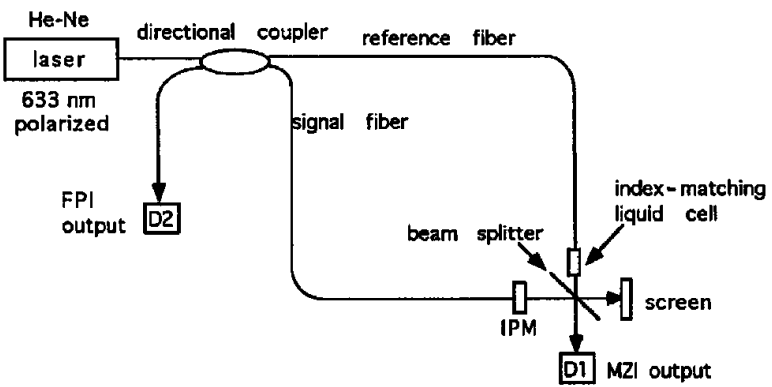

Fig. 2. Combined MZI-FPI configuration for the measurement of dynamic phase shift. 
This is because the two backreflected beams arising from the ITO surfaces travel the same distance within the signal fiber before reaching D2, resulting in lead insensitivity to phase drifts by common path rejection.

To overcome the effect of random phase drifts on the MZI, which is sensitive all along the path from the directional coupler to the beam splitter, we enclosed the whole setup within a box that cut off air currents. The combined MZI-FPI configuration utilized here permitted the direct verification of whether the intensity modulation seen at D1 and D2 was truly caused by the phase shift induced as a result of the strain generated at the piezofilm. The phase shift as measured at D2 should be twice that as measured at D1, because the reflected signal-modulated beam in the direction of D2 traverses the piezofilm twice, whereas the transmitted signal-modulated beam in the direction of D1 transverses the piezofilm once. The transmission FPI output in the direction of D1 was neglected because experiments showed that the signal arising from this was far too small in relation to that from the transmission MZI output. This is reasonable because the output from the fiber diverges as it crosses the piezofilm, which on multiple-beam interference in different directions results in an averaged time-dependent intensity within the collection cone of the detector. It is also well known that the FPI has a poor contrast in the transmission mode in comparison with that in the reflection mode. It should be noted ${ }^{9}$ that a special fringe pattern was obtained and utilized at D1, whereas the output at D2 is a zero-order fringe that has no spatial modulation.

The outputs from photodetectors D1 and D2 were amplified and fed into a digital oscilloscope that performed a fast Fourier transform (FFT) to provide a frequency spectrum of the photovoltage. Alternatively, the time-domain output recorded by the digital oscilloscope can be imported into a computer that can then perform the filtering, FFT, and the calculations for the phase shift. For the purpose of determining the frequency response or the linearity of response when the phase shift induced is not more than $0.3 \mathrm{rad}$ (which can be estimated by observing and studying the peaks in the frequency spectrum), it would suffice to plot the photovoltage, $V(f)$, at the fundamental frequency as a function of the input frequency or the voltage applied to the piezofilm. As no feedback for stabilization was applied to the interferometer, the maximum value of $V(f)$ over a prolonged period of time was measured for each input voltage or each input frequency. Over such an extended period of time, the random phase drift would scan the whole range of 0 to $2 \pi \mathrm{rad}$ and attain a value of $\pi / 2$ several times, which corresponds to the maximum value for $V(f)$. Under the approximation that the value of the Bessel function of the first order is proportional to its argument, the plot of $V(f)$ as a function of the input voltage would determine the linearity of response at a chosen frequency, whereas the plot of $V(f)$ as a function of frequency would determine the frequency response of the modulator.

When the phase shift induced is appreciable as seen from the surfacing of the higher harmonics in the frequency spectrum, the $J_{1} \ldots J_{4}$ method $^{2}$ can be implemented to measure the phase shift directly. The $J_{1} \ldots J_{4}$ method utilizes the photovoltage amplitudes at the fundamental frequency $[V(f)]$ and the next three harmonics [namely, $V(2 f), V(3 f)$, and $V(4 f)]$ through the Bessel recurrence relation to provide a linear, direct, and self-consistent readout of the dynamic phase shift in a homodyne interferometer without the need for feedback or phase biasing at the quadrature point, source stabilization, and fringe visibility control.

\section{B. Detection of Polarization Modulation}

Inserting polarization-sensitive optical elements and modifying the arrangement shown in Fig. 2 would permit the concurrent detection of polarization modulation and phase modulation. Thus, for a set of experiments that involved such concurrent detection, the FPI mode for interferometric phase detection and the transmission polarimetric mode for polarization detection were utilized as shown in Fig. 3(a). The output of the reference fiber terminated in indexmatching liquid was not used, unlike in Fig. 2, and the beam splitter was removed so that the MZI output was absent. In a different set of experiments, the fiber directional coupler was not utilized and a typical transmission ellipsometric arrangement consisting of a serial placement of a half-wave plate, the IPM, an optional quarter-wave plate, and an analyzer was set up as shown in Fig. 3(b). This was done to study the behavior of the film when not attached to the fiber connector. Whereas some of the experiments were performed with a compensator, others were not; the choice depended on the achievement of maximum sensitivity of detection for a given input signal power and linearity of operation for a chosen amplifier gain.

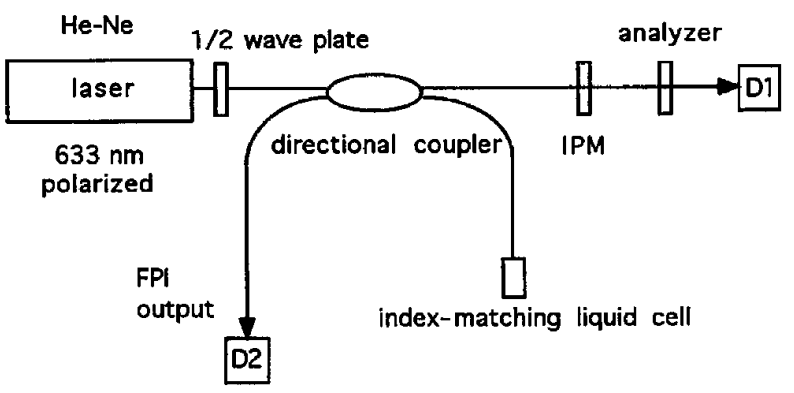

(a)

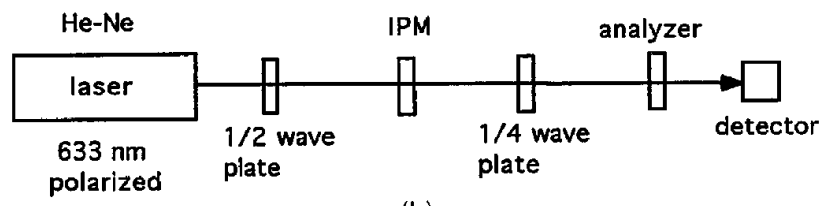

(b)

Fig. 3. (a) Extrinsic FPI and polarimetric mode of concurrent detection of phase and polarization modulation. (b) The transmission ellipsometric arrangement in the PSCA mode. 
The compensator, typically a quarter-wave plate at the wavelength of operation, was placed after the IPM, conforming to the PSCA (polarizer-samplecompensator-analyzer) arrangement. ${ }^{10}$ As a polarized laser was used, a half-wave plate was included to rotate the angle of polarization of the beam incident upon the IPM.

The phase difference between the ordinary ray and the extraordinary ray on traversing the IPM and the compensator can be expressed as ${ }^{11}$

$$
\phi=(2 \pi L / \lambda)\left(n_{e}-n_{o}\right)+\Delta \phi_{c}+\Delta \phi_{r}+\Delta \phi_{s},
$$

where $L$ is the thickness of the piezofilm, $\lambda$ is the wavelength of the light, $n_{e}$ and $n_{o}$ are the refractive indices of the extraordinary and ordinary rays, respectively, $\Delta \phi_{c}$ is the phase change on traversal through the compensator, $\Delta \phi_{r}$ is the random phase drift caused by ambient air currents and temperature fluctuations, and $\Delta \phi_{s}$ is the phase shift induced by the signal applied to the piezofilm. The contribution of the intrinsic static birefringence of the piezofilm at a fixed temperature is expressed by the first term on the right-hand side of Eq. (1). Neglecting optical loss on transmission through several elements, and on adjusting the compensator for a 50\% transmission in the absence of the modulating voltage, ${ }^{11}$ one can express the time-dependent intensity at the output of the analyzer $\mathrm{as}^{2}$

$$
\begin{aligned}
I= & \left(I_{0} / 2\right)\left(1-\cos \Delta \phi_{R}\left[J_{0}(m)+2 \sum_{n=1}^{\infty} J_{2 n}(m) \cos (2 n \omega t)\right]\right. \\
& \left.+\sin \Delta \phi_{R}\left(2 \sum_{n=1}^{\infty} J_{2 n-1}(m) \sin [(2 n-1) \omega t]\right)\right)
\end{aligned}
$$

where $I_{0}$ is the average intensity of the light incident upon the detector, $J_{n}$ 's are the Bessel functions of the first kind and order $n, m$ is the depth of polarization modulation, and $\omega$ is the angular frequency of the signal.

The phase term caused by static birefringence in Eq. (1) has been lumped with the phase terms caused by the compensator and the random drifts to form a total phase term, $\Delta \phi_{R}$, in Eq. (2). It must be pointed out that although Eq. (2) bears a close resemblance to the expression for the instantaneous intensity in an interferometer, the intensity modulation can be observed only if polarization-sensitive elements are present. The physical mechanism by which the polarization modulation represented by depth $m$ is brought about is discussed in Subsection 3. E. In comparison with the interferometric arrangement, the effect of random phase drifts in the polarimetric arrangement is relatively smaller. However, the existence of a slow drift far less than that generally observed in the MZI setup was observed even after enclosure within a box that cut air currents off. It is possible that this slow drift arose from the temperature dependence of the film birefringence, as the PVDF material is known to be a highly sensitive pyroelectric detector. It must be pointed out that the temperature of the piezofilm was not controlled by any feedback mechanism, as is commonly done ${ }^{11}$ for single-crystal modulators. Under the assumption that the value of modulation depth $m$ is small, the output voltage at the fundamental frequency would be proportional to $m$ when the condition $\Delta \phi_{R}=\pi / 2$ is fulfilled, thus facilitating the measurement of both the linearity of response and the frequency response. However, the value of $m$ cannot be determined from the amplitude at the fundamental frequency alone unless the exact instantaneous values of the intensity, visibility, and phase drift are known.

The close resemblance of Eq. (2) to the instantaneous intensity of an interferometric system suggested the possibility of utilizing the $J_{1} \ldots J_{4}$ method $^{2}$ for the direct, linear measurement of the polarization modulation depth, $m$. This is the first time that the $J_{1} \ldots J_{4}$ method has been applied to such detection of polarization modulation, the applications hitherto having been to the measurement of the dynamic phase shift in an interferometer. The measurement of $m$ through the $J_{1} \ldots J_{4}$ method directly led to the determination of the half-wave voltage, $V_{\pi}$, at which the polarization modulation depth reaches a value of $\pi$ rad. As any kind of bonding was observed to constrain the piezofilm and reduce the PSC, the free-standing piezofilm configuration of the IPM was most suitable for generating a polarization modulation large enough to be detected by the $J_{1} \ldots J_{4}$ method. The structural design and optical arrangement of this and other configurations of the IPM are described below.

\section{Structural Arrangement of the IPM}

Several configurations of the IPM that differed in the clamping conditions, the utilization of epoxy, and the interferometric arrangement were studied and characterized for the device performance. The clamping conditions and the optical arrangement for testing these IPM elements are summarized in Table 1. For convenience of reference, the acronym IPM for the four IPM elements is followed by a letter A, B, C, or D to distinguish between the individual elements. Figure 4 shows the geometry of the arrangement for the IPM elements for coupling light in general from the fiber output or directly from the laser source to the piezofilm. The cross-sectional view of the IPM is shown in Fig. 4(a), whereas the direct end-face view is shown in Fig. 4(b). The piezofilm with wire leads attached at its edges was sandwiched with the use of an epoxy between two identical circular metal washers with the dimensions indicated in Fig. 4(b). The purpose of using a metal washer was to have the boundary clamping conditions well defined in geometry and stresses. It has been reported ${ }^{3,4}$ that the frequency response and PSC of the piezofilm-based modulators are sensitive to the mechanical forces acting on them because of the wire lead attachments and holding forces at their boundary with the mounting arrangement. The use of a circular metal washer 


\begin{tabular}{|c|c|c|c|c|}
\hline $\begin{array}{c}\text { Element } \\
\text { IPMA }\end{array}$ & $\begin{array}{l}\text { Clamping Conditions } \\
\text { Circular washer on both sides of the } \\
\text { piezofilm }\end{array}$ & $\begin{array}{l}\text { Coupling Arrangements } \\
\text { Fiber ST connector butt-coupled or jux- } \\
\text { taposed to piezofilm }\end{array}$ & $\begin{array}{l}\text { Optical Configuration } \\
\text { MZI-FPI; polarimetric }\end{array}$ & $\begin{array}{c}\text { Quarter-Wave } \\
\text { Plate Used } \\
\text { No }\end{array}$ \\
\hline IPMB & $\begin{array}{l}\text { Circular washer on both sides of the } \\
\text { piezofilm }\end{array}$ & $\begin{array}{l}\text { Fiber ST connector epoxied to piezo- } \\
\text { film }\end{array}$ & FPI; polarimetric & No \\
\hline IPMC & $\begin{array}{l}\text { Circular washer on only one side of the } \\
\text { piezofilm }\end{array}$ & No fiber used; direct laser beam & Polarimetric & Yes \\
\hline IPMD & $\begin{array}{l}\text { Rectangular film freely supported on a } \\
\text { glass slide }\end{array}$ & No fiber used; direct laser beam & Polarimetric & Yes \\
\hline
\end{tabular}

led to a repeatable characterization of the modulator performance.

After the epoxy dried, the inner metal washer was epoxied to a larger rubber ring, and this whole arrangement was mounted on a rotary stage. The fiber in the signal arm was terminated in an ST (a GTE trademark) connector and polished to achieve a smooth and flat end face. In the case of interferometric phase measurements for IPMA, the ST connector mounted on a micropositioner was butt-coupled to the piezofilm, forming a small depression in the film from applied stress. In the detection of polarization modulation, the ST connector was juxtaposed to the piezofilm without any physical contact. The difference in the arrangement permitted the study of the frequency response in the loaded and unloaded conditions. IPMA was tested in both the combined MZI-FPI and the polarimetric configurations. IPMB differed from IPMA in that the ST connector was directly bonded to the piezofilm with an optically transparent epoxy. This was done to study the loading effect of the epoxy in practical conditions, in which an arbitrary distance between the fiber output and the piezofilm may not be desirable, necessitating a well-defined, repeatable contact. A photograph of IPMB is shown in Fig. 5, with electrical contact between the wire leads and the piezofilm being established through thin, flexible, copper foil backed by a thick paper. IPMB was tested in the FPI and polarimetric configurations simultaneously, thus providing an instantaneous comparison of the intensity modulation arising from the interferometric and polarimetric outputs. IPMC had a metal washer on only the inner side of the film, not on the outer side, with the mounting on the rotary stage the same as in the case of IPMA. The beam from the laser source was used directly as shown in Fig. 3(b), with only the polarization modulation having been of interest. The optical fiber was not used for transmission as in IPMB, because once the piezofilm is epoxied to the fiber ST connector at a particular angle, the IPM should not be rotated to align its fast and slow axes to a different angle. This limitation can be overcome if a polarization-preserving fiber is used that is insensitive to twists and bends.

The fiber used in our experiments was a circular core single-mode fiber whose birefringence was dependent on external twists and bends. IMPD consisted of a piezofilm cut in a rectangular shape with a tail leading to the wire leads for electrical contact, as shown in Fig. 4(c). The film was not bonded at any point, thus eliminating any stresses from the supporting thick $(1-\mathrm{mm})$ glass slide. As the natural residual curvature of the piezofilm was directed toward the glass slide, the film was freely supported in a stable position by the holding forces at the tail section. The purpose of testing this IMPD was to generate a polarization modulation that was strong enough that the $J_{1} \ldots J_{4}$ method could be utilized for measuring $m$ and thereby the half-wave voltage, $V_{\pi}$. IPMD was tested with the beam from the laser source being directly incident upon the piezofilm without the use of an optical fiber. It should be emphasized that the size of the piezofilm forming the IPM need not exceed the dimensions of the fiber itself or the spot size of the laser beam itself. This enhances the significance of the possibility of depositing the electrodes and the PVDF film directly on the fiber itself. Although such a fabrication would involve sophistication, the device would be compact and compatible with standard fiber-optic components currently used in the industry. However, in the research reported here, the piezofilm was prefabricated, requiring the use of a larger area of the piezofilm because of practical considerations for laboratory purposes.

\section{Results and Discussion}

\section{A. Interferometric Phase Detection}

The induced phase shift measured by the $J_{1} \ldots J_{4}$ method as a function of the input voltage applied to IPMA in the FPI mode at $2 \mathrm{kHz}$ is shown in Fig. 6. The least-squares fit to the plot shown in Fig. 6 was determined to be $\phi=-0.0004$ voltage peak squared $\left(\mathrm{Vp}^{2}\right)+0.131 \mathrm{Vp}+0.0008 \mathrm{rad}$, indicating a linear PSC of $0.131 \mathrm{rad} / \mathrm{Vp}$ at $2 \mathrm{kHz}$. Figure 7 shows the frequency response measured in the FPI mode for IMPA. The output voltage amplitude at the fundamental frequency of the signal was normalized with respect to the input voltage applied, and it was plotted as a function of the frequency of the signal. This normalization overcame the inherent change of the output voltage of the function generator itself as a function of the frequency. The normalized output exhibits a resonance peak at a frequency of $7.5 \mathrm{kHz}$. In comparison with the flat strip fiber-on-PVDF film 


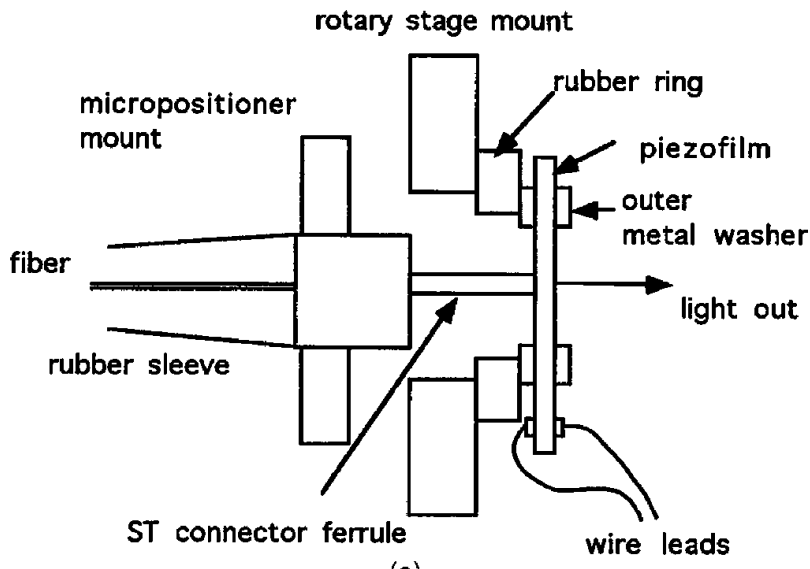

(a)

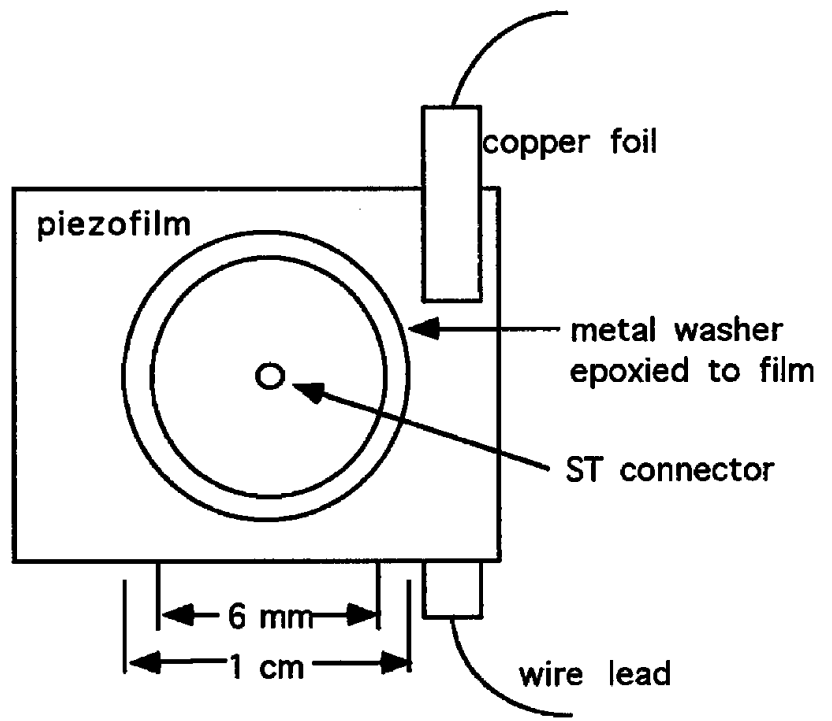

(b)

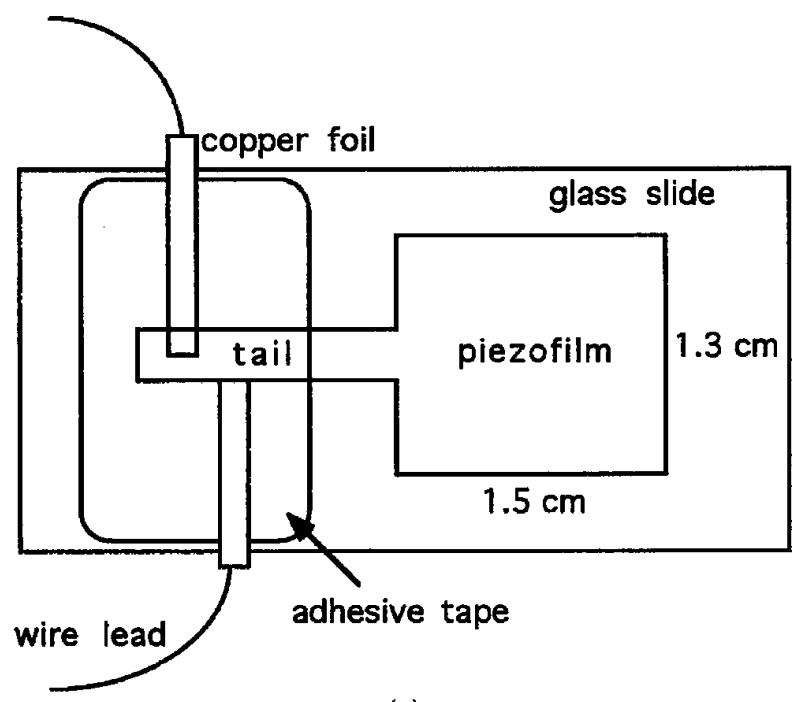

(c)

Fig. 4. General mounting arrangement for the elements IPMA, IPMB, and IPMC: (a) cross-sectional view, (b) direct end-face view. (c) Geometry of IPMD.

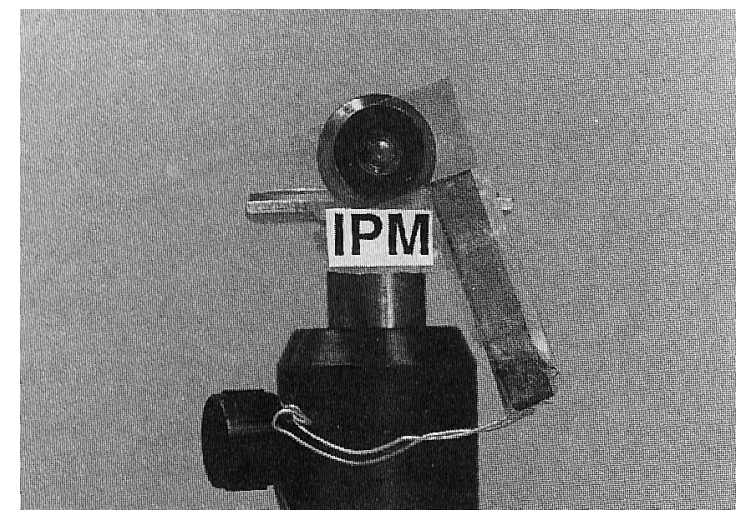

Fig. 5. Photograph of the direct end-face view of IPMB with the ST connector epoxied to the piezofilm at the center of the circular washer.

(FPF) modulator, ${ }^{3}$ which shows a resonance in the region of $3-4 \mathrm{kHz}$, this presents a better suitability to fiber-sensor applications wherein a higher frequency of operation is generally preferred to overcome lowfrequency $1 / f$ noise. That the phase shift as measured by the reflection FPI mode at D2 must be twice that measured by the transmission MZI mode at D1 was confirmed by the combined MZI-FPI configuration for IPMA.

Figure $8 \mathrm{a}$ shows the FFT frequency spectrum of the output of the MZI mode, whereas that of the FPI mode is shown in Fig. 8b. The phase shifts at $2 \mathrm{kHz}$ for these two modes as measured by the $J_{1} \ldots J_{4}$ method were 0.97 and $1.923 \mathrm{rad}$, respectively. This was further confirmed at the resonance frequency of $7.4 \mathrm{kHz}$ as shown for the MZI and FPI modes in Figs. $8 \mathrm{c}$ and $8 \mathrm{~d}$, respectively. The phase shifts measured at $7.4 \mathrm{kHz}$ from these plots were 1.67 and $3.23 \mathrm{rad}$ for an input voltage of $6.57 \mathrm{Vp}$. Thus, the fact that the phase shift originated from the integrated strain generated in the piezofilm and not from any out-ofplane body displacement of the piezofilm in the direction of the light beam is confirmed.

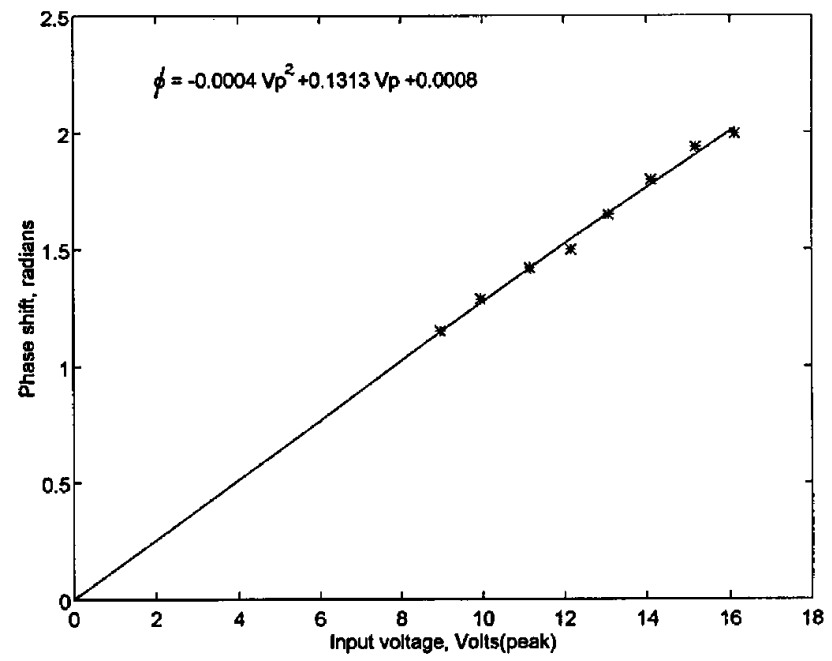

Fig. 6. Phase shift measured by the $J_{1} \ldots J_{4}$ method as a function of the input voltage applied to IPMA at $2 \mathrm{kHz}$. 


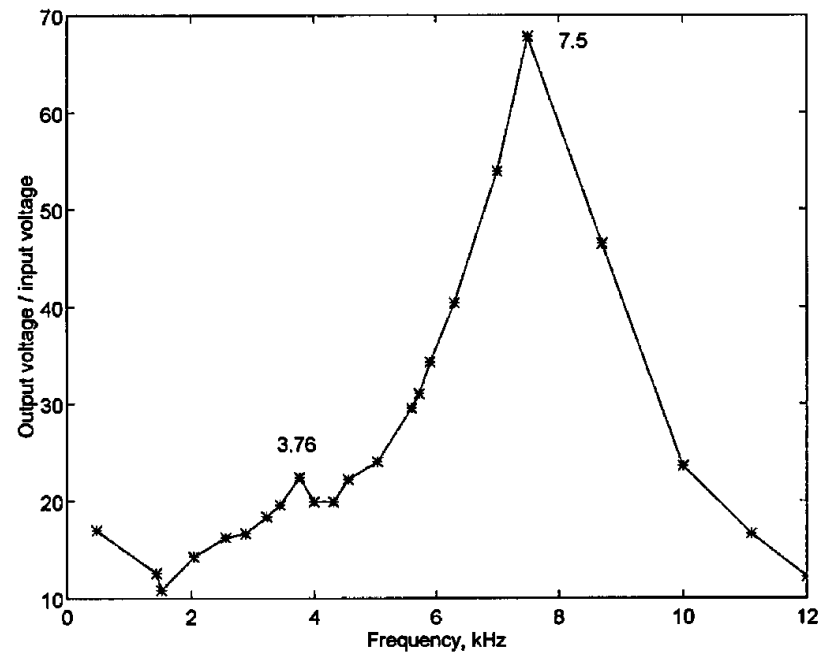

Fig. 7. Frequency response of IPMA measured in the FPI mode. Note that the curves connecting the data points are only for indication and are not the best-fit curves.

Further, as the resonance frequency is reached the PSC becomes larger, with the result that the PSC at $7.4 \mathrm{kHz}$ was $0.508 \mathrm{rad} / \mathrm{Vp}$. The PSC of $1.77 \mathrm{rad} / \mathrm{Vp}$ for the flat strip FPF modulator ${ }^{2,3}$ reported earlier was obtained through the utilization of $1 \mathrm{~m}$ of interaction length of the fiber. In comparison with this length, the interaction length in the IPM is the thickness of the piezofilm itself, only $63.9 \mu \mathrm{m}$, as measurements reported in the next subsection show. Comparing these values, we see that the performance of the IPM direct modulation arrangement is far superior to that of the flat strip FPF modulator. Also, a single piezofilm has been used here, whereas a stack of films bonded together with an optically transparent epoxy can be used to multiply the phase shift produced. For this purpose, it would also be worthwhile to deposit a multilayer element consisting

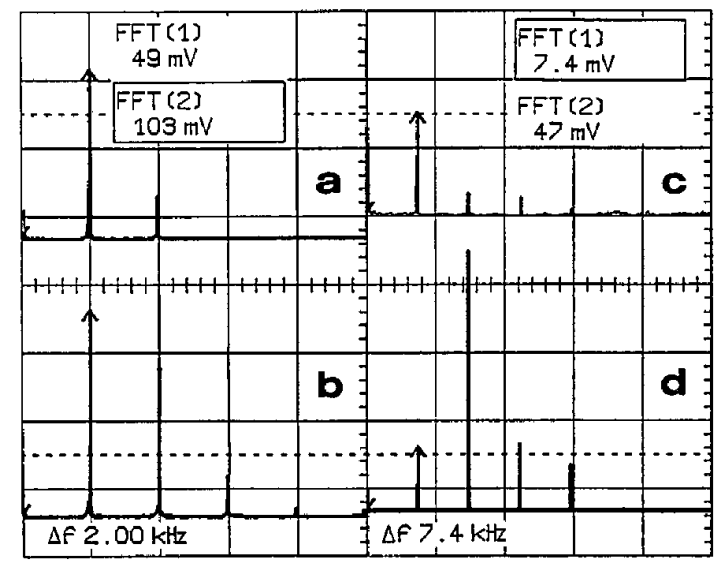

Fig. 8. FFT of the output of a, the MZI and b, the FPI for phase shift at $2 \mathrm{kHz}$, and c, the MZI and d, the FPI for phase shift at the resonance frequency of $7.4 \mathrm{kHz}$. The vertical axis in each of these plots indicates the photovoltage, whereas the horizontal axis indicates the frequency. of alternate films of PVDF and ITO to avoid the use of epoxy.

\section{B. Piezofilm Birefringence and Thickness Measurement}

For the study and understanding of the polarization modulation in the presence of an applied electric field, it is necessary to know (a) the intrinsic static birefringence of the piezofilm in the absence of the electric field, (b) the thickness of the piezofilm for characterizing the PSC and the depth of polarization modulation, and (c) the directions of the fast and slow axes of the film for achieving proper orientation of the film with the other optical elements. The polarization eigenaxes of the piezofilm can be determined through the use of the transmission ellipsometric arrangement shown in Fig. 3(b) without the quarter-wave plate. The half-wave plate and the analyzer are rotated alternately until the intensity seen at the detector on transmission through the piezofilm is minimum. Thus, one would find two mutually perpendicular directions of the piezofilm for which the minimum intensity occurs. The piezofilm as provided by the manufacturer would show visible macroscopic straight lines on the film surface as a result of the uniaxial stretching necessary to make the polymer film piezoelectric. ${ }^{12-14}$ The eigenaxes of the piezofilm are thus seen to occur naturally in the directions along the stretch and perpendicular to it. In practice, the straight-line stretch marks generate a scattering pattern, which is a thin straight band again when an uncollimated beam of small diameter is used directly from the laser. This thin band of scattered light, seen in both the forward and backward directions of propagation, would be perpendicular to the stretch direction and is used for quick alignment of the piezofilm.

We measured the optical birefringence of the piezofilm by using the automated Metricon 2010 prism coupler. The principle of operation of the prism coupler ${ }^{15}$ closely resembled that of an Abbe refractometer. The piezofilm of refractive index $n$ was brought in close contact with the base of a prism of refractive index $n_{p}$. The light from a He-Ne laser operating at $633 \mathrm{~nm}$ was incident upon the base of the prism, making an angle, $\theta$, with the normal to the prism base at the point of incidence. This beam would be totally reflected into the prism when the critical angle, $\theta_{c}$, is reached, where

$$
\theta_{c}=\sin ^{-1}\left(n / n_{p}\right) .
$$

The critical angle is directly measured by the observation of the output of a photodetector that accesses the reflected beam coming out of the prism. The detector output drops abruptly as $\theta$ becomes less than $\theta_{c}$ when light begins to travel into the film. With the value of $n_{p}$ known, refractive index $n$ of the film can be determined from Eq. (3). For a thick film as is the case here, an approximation is made that the angle of the first film mode equals the critical angle. The error in the measured index caused by this approximation is typically less than 0.004 for a film thickness of 
$3 \mu \mathrm{m}$, less than 0.001 at $5 \mu \mathrm{m}$, and less than 0.0003 at $10 \mu \mathrm{m}$.

When the laser emits polarized light, TE incidence (electric field vibrating transverse to the plane of incidence) and TM incidence (electric field parallel to the plane of incidence) can be used to determine the refractive indices in the plane of the film and perpendicular to it, respectively. In the plane of the film, under TE incidence, one can find the refractive indices in the directions of the fast and slow axes by rotating the film appropriately. As we mentioned above, the eigenaxes of the piezofilm are along and perpendicular to the stretch marks. Figure 9 shows the output of the detector for the determination of the index along the stretch marks, under TE incidence. By locating the angle at which the knee in the intensity occurs and by using Eq. (3), we determined the refractive index to be 1.4452 along the stretch marks. Rotating the piezofilm in its plane by $90^{\circ}$ and repeating the measurement led to the determination of the index perpendicular to the stretch marks of 1.4124. For the determination of the index in the direction perpendicular to the plane of the film, TM incidence was used, leading to a value of 1.4112 . Thus a birefringence of 0.034 was measured between the directions of the stretch and the perpendicular to the plane of the film, whereas a birefringence of 0.0328 was measured between the directions of the stretch and its perpendicular both in the plane of the film. These values were repeatable as observed in a self-consistency check involving several trials. These values are also in close agreement with those reported earlier ${ }^{16}$ for bare PVDF film with no electrodes on it. The necessity for the exact measurements in this study arose because our purpose was to characterize the piezofilm with the ITO electrodes present as would be used in our device, and because the piezo film was obtained from a manufacturer different from that for the earlier reports. It must be noted that

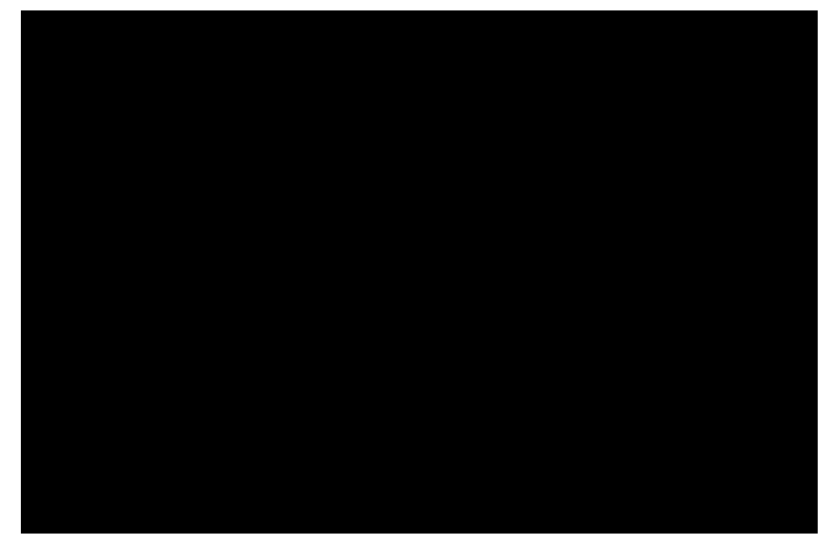

Fig. 9. Variation of the totally reflected intensity seen by the detector in the prism coupler as a function of the angle of incidence of the laser beam. The numbers on the horizontal axis correspond to discrete steps in angle, with 20 steps $=1^{\circ}$ and the reference direction of $0^{\circ}$ being along the perpendicular to the prism incident face. This plot is for TE incidence along the direction of the stretch marks on the piezofilm. the ITO layers have a high value of refractive index ${ }^{17}$ of approximately $1.9+i 0.025$. However, as we can see on comparing the measurements of this study and that of Ref. 16, the ITO layers did not influence the obtained index values appreciably because the thickness of the ITO was far smaller in relation to that of the PVDF layer.

It is possible to utilize the transmission spectrum of a weakly absorbing thin film to measure its refractive index and thickness, as reported in Ref. 18. Such a method considers the envelopes of the maxima and minima of transmittance as continuous functions of the wavelength. The method would be accurate only if the transmittance values of the maxima and minima are largely different for the wavelength of interest, and if the variation with wavelength of the refractive index and absorption coefficient is small. As seen from Fig. 1, both of these conditions were not fulfilled in the short-wavelength region in which the $\mathrm{He}-\mathrm{Ne}$ laser (633-nm) light used in our experiments lay. However, an estimate of the thickness, namely $62 \mu \mathrm{m}$, was obtained from the long-wavelength region in which sharp fringes are seen under the assumption that the refractive index is constant over a period of two maxima. To overcome this limitation of the method to our present study, we utilized a variable angle monochromatic fringe observation method. ${ }^{19}$ This method utilizes the interference maxima and minima in the light reflected from the film surfaces as the angle of incidence of the light is varied. Because a single wavelength is used, which in our experiments was $633 \mathrm{~nm}$, the measurement does not require knowledge of the refractive index as a function of the wavelength except that at the single chosen wavelength. The values of the refractive indices already measured at $633 \mathrm{~nm}$ by the critical angle method thus were used to determine the thickness accurately. Figure 10 shows the maxima and the minima for the PVDF film with the ITO electrodes. The expression for the thickness, $d$, of the film is given by ${ }^{19}$

$$
d=\Delta N \lambda /\left[2 \mu\left(\cos r_{2}-\cos r_{1}\right)\right],
$$

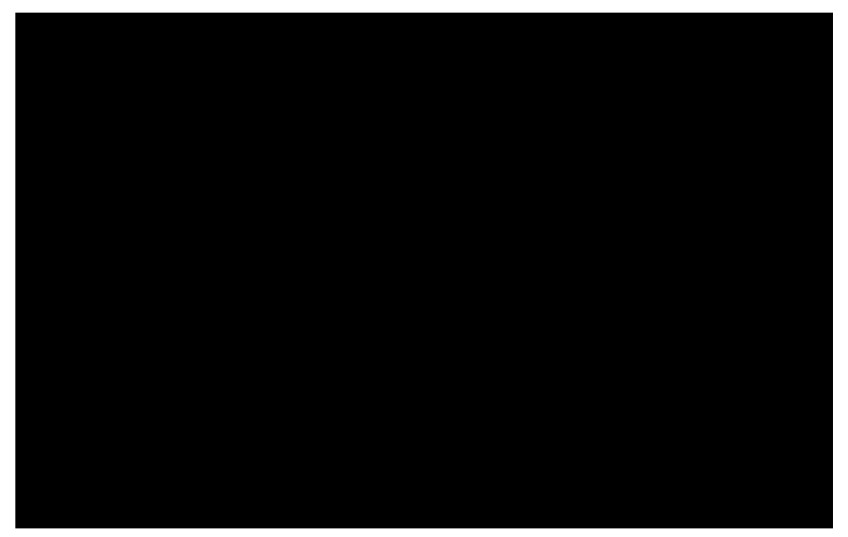

Fig. 10. Variation of the surface-reflected intensity seen by the detector in the variable angle monochromatic fringe observation arrangement as a function of the angle of incidence of the laser beam. The numbers on the horizontal axis indicate discrete steps in angle, with 40 steps $=1^{\circ}$. 
where $\lambda$ is the wavelength, $\mu$ is the refractive index of the film, $r_{j}$ is the angle of refraction at that fringe for which the angle of incidence is $i_{j}$ and $\sin r_{j}=$ $\left(\sin i_{j}\right) / \mu$, and $\Delta N$ is the number of fringes observed between $i_{1}$ and $i_{2}$. Using this expression in an averaged form, ${ }^{19}$ we determined the thickness of the piezofilm used in the IPM to be $63.93( \pm 1.74) \mu \mathrm{m}$.

\section{Polarization Modulation Measurement}

IPMA was tested in the polarimetric mode after blocking the output from the reference fiber as seen in Fig. 2 and inserting a half-wave plate and analyzer as shown in Fig. 3(a). The ST connector was moved away from the piezofilm as mentioned earlier. An input voltage at the frequency of $8.4 \mathrm{kHz}$ was given to IPMA. Figure 11 shows the output of IPMA as seen at the detector as the analyzer is rotated for an arbitrary angle of the incident polarization. Such an intensity modulation was as expected, showing that the signal seen at $8.4 \mathrm{kHz}$ was due to polarization modulation. A similar type of intensity variation was recorded as the half-wave plate was rotated and is also shown in Fig. 11. As we expected, the period for the half-wave plate rotation was half that for the analyzer rotation. One could use these plots for choosing the angles of the half-wave plate and the analyzer such that the modulation depth is optimized for a given relative angle of the IPM element. Whereas the intensity modulation seen in Fig. 11 arose from polarization modulation, concurrent phase modulation could also lead to intensity modulation if reflection-based interference effects exist.

To evaluate this effect, we recorded the instantaneous intensity at the detector (which had an effective aperature of $1 \mathrm{~cm}^{2}$ ) as the analyzer was brought in place and subsequently removed. As we see from Fig. 12, the detector output showed a signal at 8.4 $\mathrm{kHz}$ in the presence of the analyzer between the start

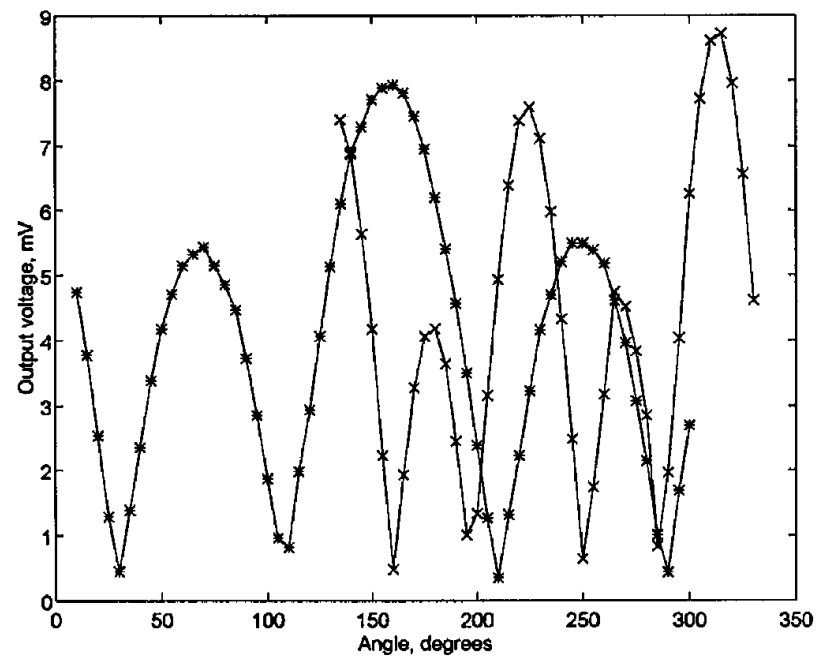

Fig. 11. Output of IPMA as seen by the detector when the analyzer is rotated $\left(^{*}\right)$ for an arbitrary angle of the half-wave plate, and the angle of the half-wave plate is rotated $(\times)$ for an arbitrary angle of the analyzer. Note that the curves connecting the data points are only for indication.

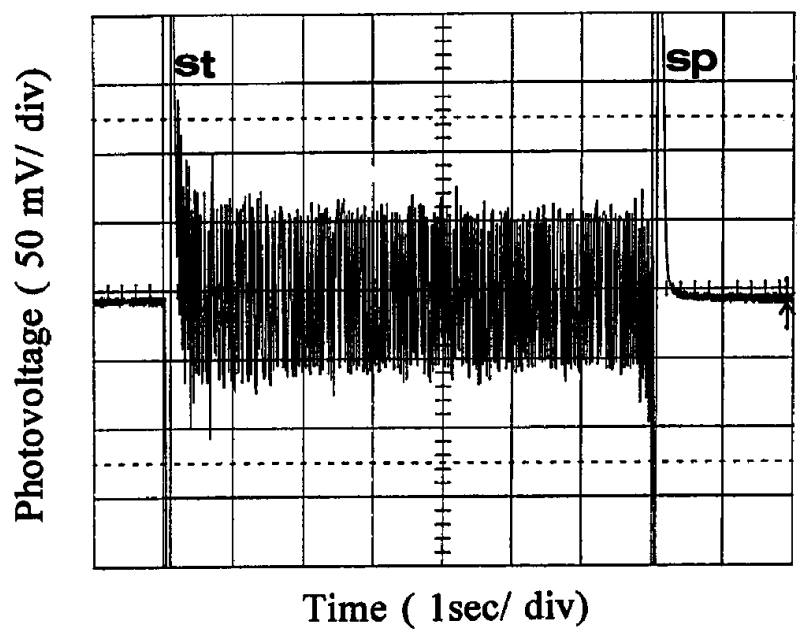

Fig. 12. Instantaneous output of IPMA when the analyzer is inserted (st) and subsequently removed (sp).

(st) and the stop (sp) points. This signal died down to a negligible level in the absence of the analyzer, demonstrating that the intensity modulation seen is indeed entirely due to polarization modulation and is not due to any reflection-based interference effects. The frequency response of IPMA as measured by the detection of polarization modulation is shown in Fig. 13 in terms of the ratio of the output voltage to the input voltage. It is seen that the significant resonance peak is shifted to $8.4 \mathrm{kHz}$ as compared with that of $7.4 \mathrm{kHz}$, as shown in Fig. 7. This change in the resonance frequency between the loaded and unloaded conditions of the piezofilm was as expected. Figure 14 shows the variation of the output voltage at the fundamental frequency of $8.4 \mathrm{kHz}$ as a function of the input voltage. The least-squares fit to this plot was determined to be $\left(\mathrm{Vo}=0.001 \mathrm{Vp}^{2}+0.644\right.$ $\mathrm{Vp}+0.176$, showing that the linearity of polarization modulation was excellent However, a direct

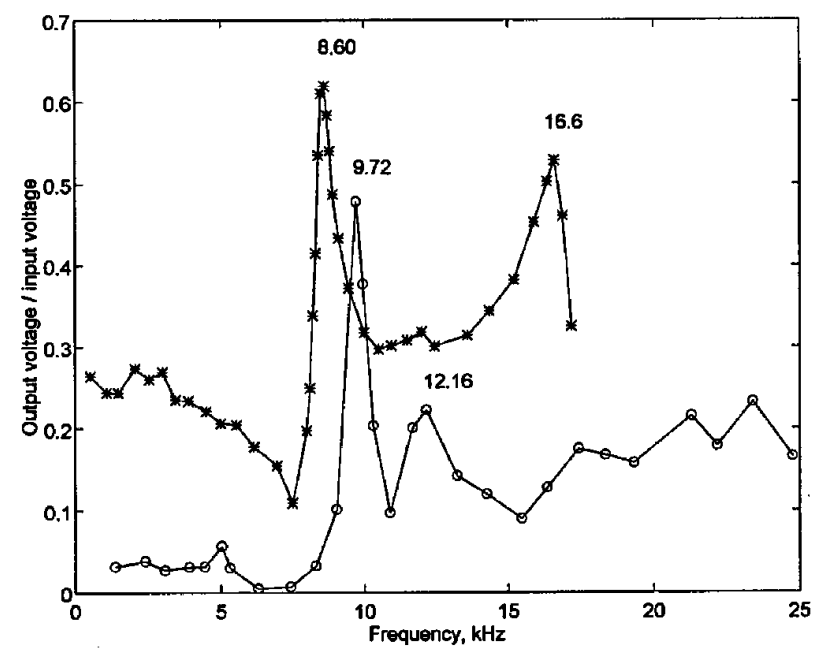

Fig. 13. Frequency response of IPMA (upper curve) and IPMC (lower curve) as measured by the detection of polarization modulation. Note that the curves connecting the data points are only for indication. 


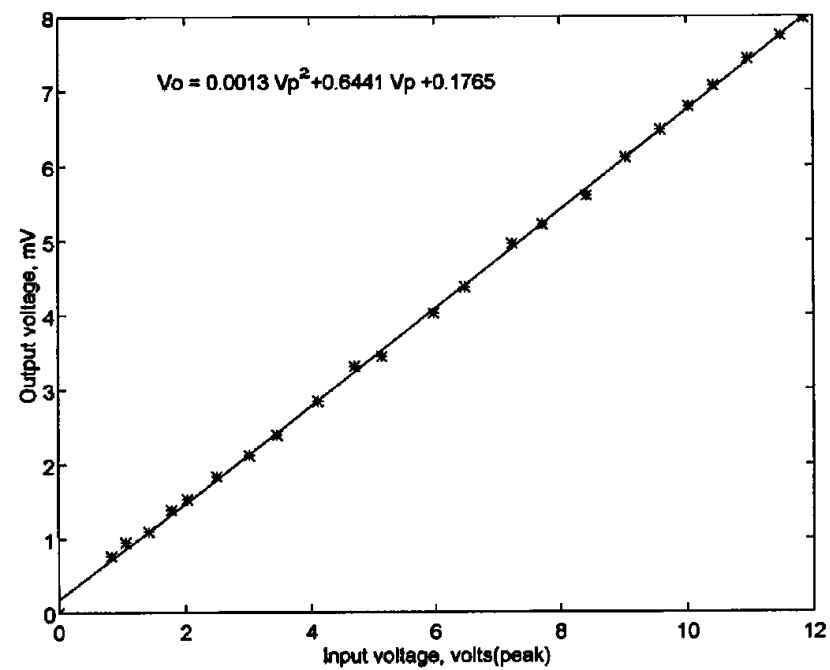

Fig. 14. Linearity of response of IPMA with the input voltage at $8.4 \mathrm{kHz}$.

measurement of modulation depth $m$ was not possible because no higher harmonics were present, indicating a very small value for $m$.

IPMB was tested in the extrinsic FPI mode and the polarimetric mode simultaneously with the experimental arrangement shown in Fig. 3(a). The linearity of response for IPMB in terms of the output voltage as a function of the input voltage at a frequency of $6 \mathrm{kHz}$ is shown for both the FPI and polarimetric modes in Fig. 15. The least-squares fit to these plots were $\left(\mathrm{Vo}=-0.041 \mathrm{Vp}^{2}+2.702\right.$ $\mathrm{Vp}+0.544)$ and $\left(\mathrm{Vo}=-0.069 \mathrm{Vp}^{2}+8.292 \mathrm{Vp}+\right.$ 0.266 ), respectively. In comparison with the results for IPMA, a higher ratio of the nonlinearity coefficient to the linearity coefficient is seen for IPMB for a similar range of input voltage. This is in agreement with the reports ${ }^{3,4}$ for the earlier piezofilm modulators, in which it was shown that the epoxy leads to a large nonlinearity in the response.

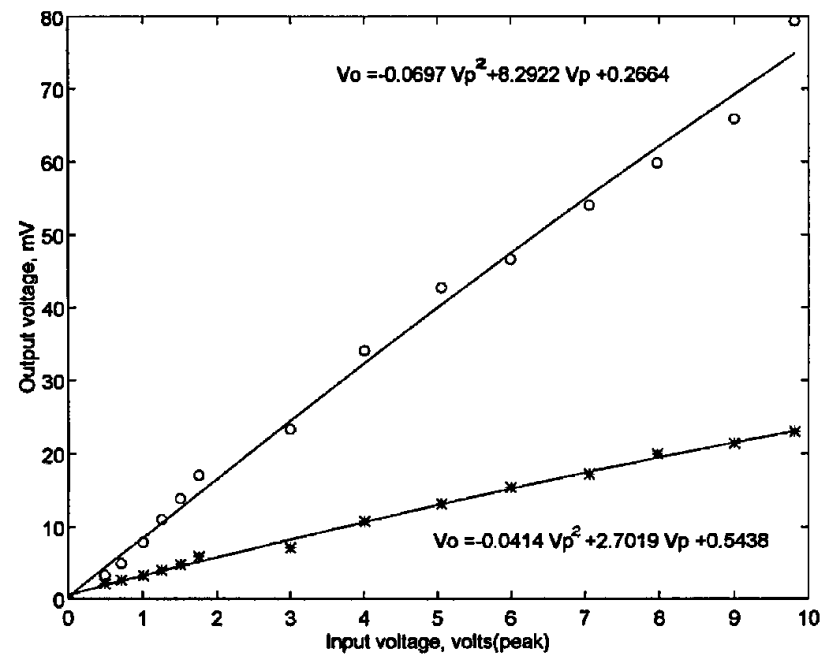

Fig. 15. Linearity of response of IPMB in the FPI mode ${ }^{*}$ ) and the polarimetric mode $(O)$ simultaneously measured with the input voltage at $6 \mathrm{kHz}$.

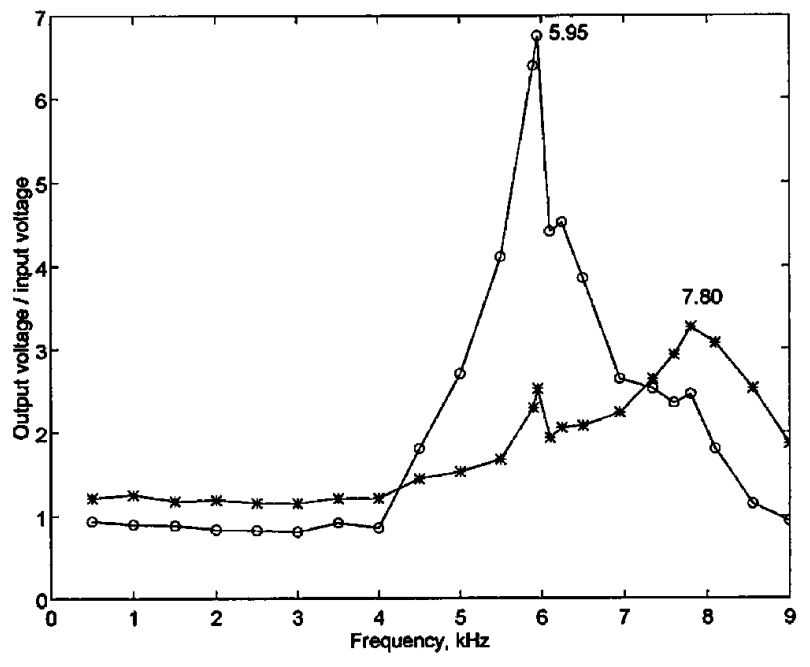

Fig. 16. Frequency response of IPMB in the FPI $\left.{ }^{*}\right)$ mode and the polarimetric mode $(O)$ for an input voltage of $10.3 \mathrm{Vp}$. Note that the curves connecting the data points are only for indication.

The frequency response of IPMB in both the extrinsic FPI and polarimetric modes is shown in Fig. 16 for an input voltage of $10.3 \mathrm{Vp}$. The two plots shown in Fig. 16 are similar in behavior, with two significant resonance peaks seen to occur in both the plots at frequencies of 5.95 and $7.8 \mathrm{kHz}$. This agreement is as expected, for the frequency-response behavior is based on the physical parameters and conditions of the device and not on the mode of detection. It should be pointed out that the detection of phase modulation utilizes the phase difference between the reference and signal arms or paths of the interferometer, whereas the detection of polarization modulation utilizes the phase difference between the orthogonal polarizations. Thus these two modes of detection would exhibit modulation characteristics that are qualitatively similar. However, the intensity modulation as seen with the polarimetric mode is strongly dependent on both the angle of the analyzer and the half-wave plate as shown in Fig. 17, whereas the FPI output is, as expected, relatively constant with the angle of the analyzer compared with that of the half-wave plate. This is because of the residual polarization sensitivity of the directional coupler, which comes into play in the interferometric mode when the half-wave plate is rotated but not when the analyzer is rotated.

IPMC was tested in the PSCA mode as shown in Fig. 3(b) with the use of the quarter-wave plate. The analyzer was crossed with respect to the direction of polarization at the exit of the half-wave plate. The eigenaxes of IPMC were aligned at $45^{\circ}$ to the analyzer and the incident polarization. As we mentioned above, the quarter-wave plate was then rotated to allow $50 \%$ transmission in the absence of the applied voltage. Figure 13 shows the frequency response of IPMC as obtained from polarization modulation. A strong resonance peak at $9.72 \mathrm{kHz}$ was observed with other appreciable peaks occurring at higher frequencies. Figure 18 shows the linearity of re- 


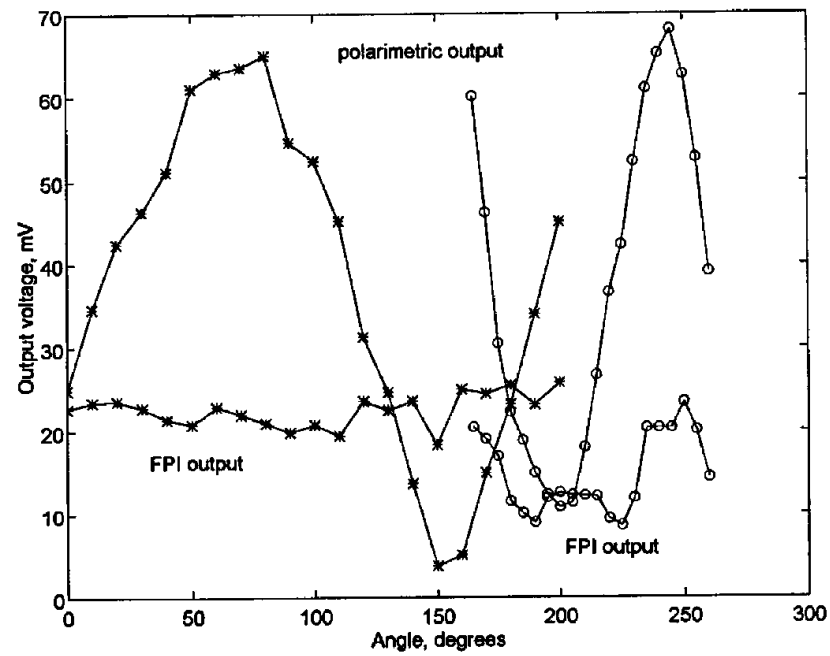

Fig. 17. Output of the FPI and the polarimetric modes for IPMB with the input voltage at $6 \mathrm{kHz}$ as the analyzer is rotated $\left(^{*}\right)$ and the half-wave plate is rotated $(O)$.

sponse for IPMC at the chosen frequencies of 9.912, $11.914,12.5$, and $17.334 \mathrm{kHz}$. This study of the linearity response at the chosen frequencies was undertaken to compare the effect of resonance on the linear behavior of the piezofilm. In contrast to the flat strip FPF modulators ${ }^{3,4}$ that showed an appreciable nonlinearity at resonance, IPMA and IPMC when not in a loaded configuration showed negligible nonlinearity even at the resonance frequencies. A comparison of the least-squares fit to these plots, shown in Fig. 18, demonstrated that the average value of the ratio of the nonlinearity coefficient to the linearity coefficient for all these plots was at least four times smaller than that for IPMB, which is an epoxied configuration.

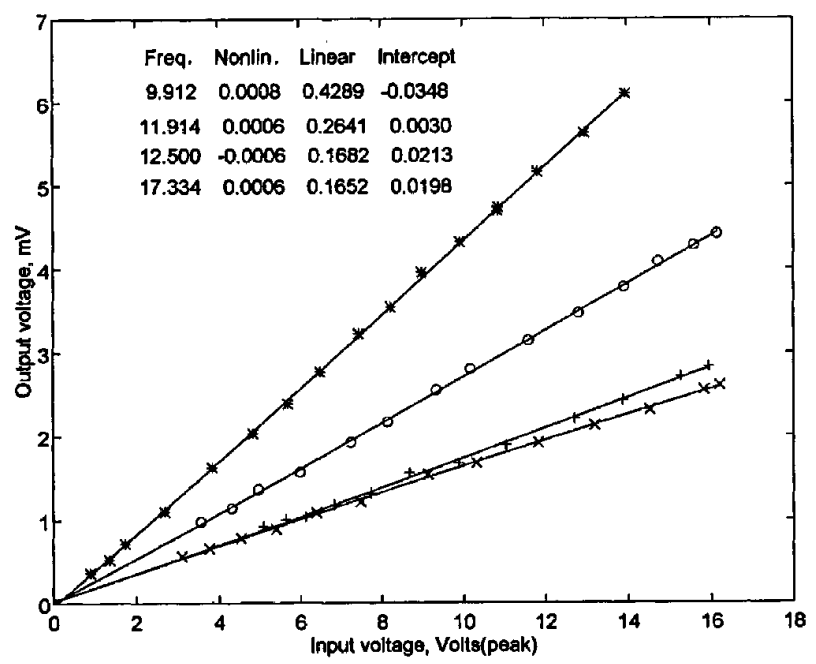

Fig. 18. Linearity of response of IPMC at the frequencies, kilohertz of $\left.9.912{ }^{*}\right), 11.914(O), 12.500(\times)$, and $17.334(+)$. The legend summarizing the least-squares fit to the plots shows the values of the frequency, the nonlinear and linear coefficients, and the intercept.

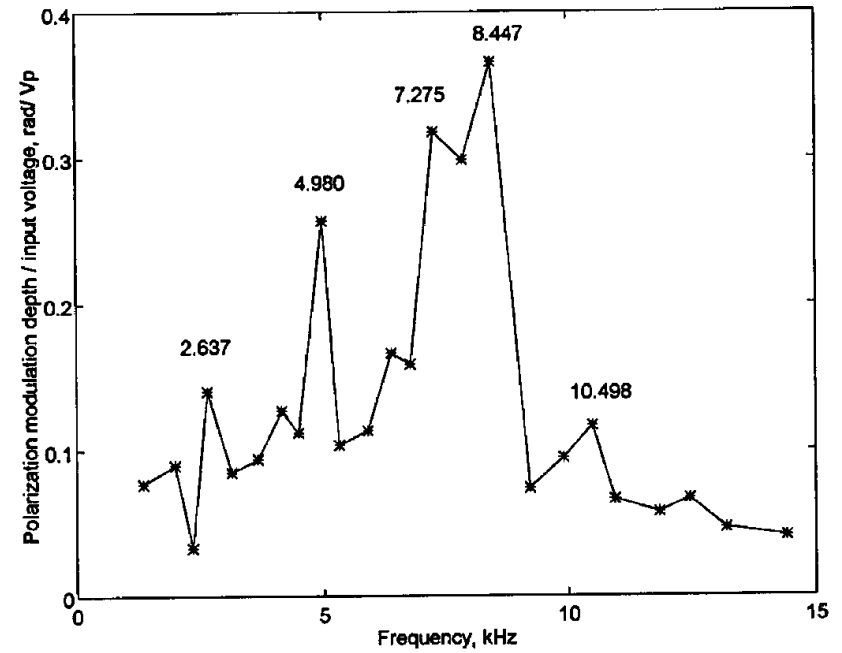

Fig. 19. Frequency response of IPMD in the polarimetric mode measured by the $J_{1} \ldots J_{4}$ method.

The freely supported piezofilm used in IPMD generated significant polarization modulation so that modulation depth $m$ could be measured by the $J_{1} \ldots J_{4}$ method. IPMD was tested in the PSCA mode as shown in Fig. 3(b). The same procedure of alignment as for IMPC was followed. Figure 19 shows the frequency response of IPMD in the polarimetric mode as measured by the $J_{1} \ldots J_{4}$ method. The depth of polarization modulation measured in radians was normalized with respect to the input voltage because the difference in the value of the phase shift for a given voltage between the resonance frequencies and others was large. The phase shift measured by the $J_{1} \ldots J_{4}$ method at $8.423 \mathrm{kHz}$ is shown in Fig. 20 as a function of the input voltage applied to the piezofilm. From the least-squares fit to this plot, shown in Fig. 20, the linear polarization modulation coefficient was found to be $0.323 \mathrm{rad} / \mathrm{Vp}$. The halfwave voltage, $V_{\pi}$, at this frequency of $8.423 \mathrm{kHz}$ (taking

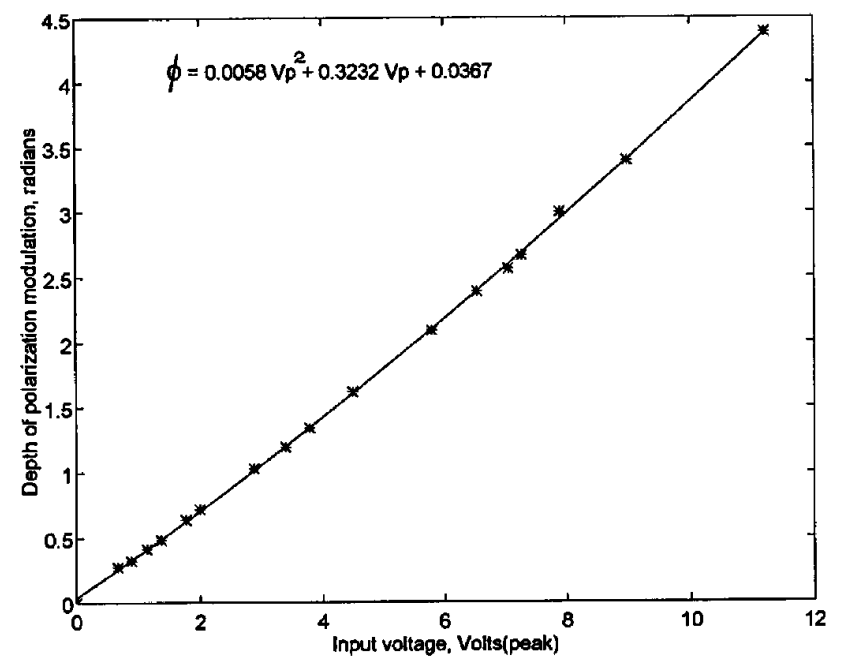

Fig. 20. Depth of polarization modulation for IPMD measured by the $J_{1} \ldots J_{4}$ method as a function of the input voltage at $8.423 \mathrm{kHz}$. 
into account the linear and nonlinear coefficients of the best fit) was determined to be $8.353 \mathrm{~V}$ (peak).

\section{Frequency Response: Implications for Design}

The behavior of the frequency response and the linearity of response for the four IPM elements seen from the above results for measurements in both the interferometric and polarimetric modes can be summarized thus: (a) the most significant resonance peak for the circularly clamped configuration in the absence of loading by the fiber ST connector occurs in the range of approximately 8.6 to $9.7 \mathrm{kHz}$; (b) when the ST connector is either butt coupled to form a depression on the piezofilm or is epoxied at the center of the piezofilm, the resonance peak shifts to a lower frequency, in the range of approximately 5.9 to $7.5 \mathrm{kHz}$, whereas (c) the freely supported IPMD shows a decrease in the modulation depth with increasing frequency after the resonance frequency, the circularly clamped configurations, IPMA, and IPMC in the absence of the ST connector (see Fig. 13) show a higher modulation depth at frequencies above the resonance peak as compared with those below the resonance peak; and (d) the inclusion of an epoxy as in IPMB results in higher nonlinearity of response. In comparison with the frequency response of the other IPM elements, the response of IPMD exhibits several significant peaks in the range of approximately 2.6 to $10.5 \mathrm{kHz}$.

Thus we can see that the circularly clamped configuration selectively enhances the response at only certain frequencies out of this range, the selected frequencies being dependent on the clamping conditions and the utilization of epoxy. The determination of the exact resonance frequencies from elasticity theory of vibrations for individual elements was not possible because the exact mechanical forces at the boundary of the elements could not be determined. The dimensions of the circular metal washer were $6 \mathrm{~mm}$ for the inner diameter and $1 \mathrm{~cm}$ for the outer diameter. In all the circularly clamped configurations, the film was bonded over the area between the inner and outer circumference. The resonance frequency for similar sample mounting in a circularly clamped configuration and similar dimensions of the metal ring, although arising from the different physical phenomenon of photothermal bending, has been reported ${ }^{20}$ to be approximately $7 \mathrm{kHz}$. The observed frequency response behavior also is an indicator of the physical mechanism that generates polarization modulation in the IPM elements, as explained in the following subsection.

\section{E. Origin of Modulation in the IPM}

The quantitative experimental results presented above were preceded by qualitative studies of the FFT of the photovoltage for the elements IPMA, IPMB, and IPMC as the input voltage was increased from 0 to a maximum of approximately $16 \mathrm{Vp}$ permitted by the function generator. The FFT showed a peak at only the fundamental frequency of the applied voltage, with no peak at the second-harmonic frequency, for even the highest voltage applied at any frequency of interest below approximately $25 \mathrm{kHz}$. This showed that the modulation arose from a linear relationship in the electro-optic interaction. IPMD showed peaks at the higher harmonics of the fundamental frequency as the input voltage was increased. However, the presence of the peak at the fundamental frequency, and its behavior as in an interferometer, established that these higher harmonics arise from the nonlinearity inherent in the relationship given in Eq. (2), and not from a quadratic electro-optic effect. Generally, electro-optic coefficients are determined at zero strain as in a sample well clamped in all directions. However, in a sample that is free to respond, the electro-optic coefficient, $r_{i j}$, measured at zero stress (freely supported) would differ from that at zero strain, $r_{i j}{ }^{\prime}$, according to the relation ${ }^{21}$

$$
r_{i j}=r_{i j}{ }^{\prime}+\sum_{k=1}^{6} p_{i k} d_{k j}
$$

where $p_{i k}$ is the strain-optic coefficient and $d_{k j}$ is the piezoelectric constant of the material.

The frequency response can then be interpreted as arising from the variation in the amplitude of the piezoelectric strain with frequency. At low frequencies, the sample is free to be strained and the value of $r_{i j}$ is obtained. At high frequencies, the strain is inhibited by the inertia of the sample, and therefore the value of $r_{i j}{ }^{\prime}$ is obtained. Thus from the frequency response studies for the different IPM elements reported above, the physical mechanism by which the polarization modulation is brought about in the IPM can be modeled as an electro-piezo-optic effect rather than as purely an electro-optic effect. The half-wave retardation voltages in the longitudinal and transverse electro-optic configurations for a PVDF film of thickness $\sim 30 \mu \mathrm{m}$ have been reported ${ }^{22}$ to be $5.6 \times$ $10^{5} \mathrm{~V}$ and $1.5 \times 10^{6}(d / L) \mathrm{V}$, respectively, where $d / L$ is the ratio between the interelectrode distance and the optical path.

As the voltages utilized here were extremely low compared with these values, and because an electric field greater than $50 \mathrm{MV} / \mathrm{m}$ is required to observe appreciable electro-optic hysteresis, ${ }^{23}$ it is reasonable to assume that the magnitude of the electro-optic effect at low voltages as utilized here is very small. The goal in the design of the IPM was to generate with the use of the PVDF film a phase and polarization modulation that was large enough at low frequencies within the upper limit of $\sim 25 \mathrm{kHz}$ for suitability to existing optical sensor systems. To this end, the piezoelectric contribution serves to enhance the device performance.

\section{Applications}

Recent interest in all-fiber devices has given rise to the development of many generic modulators based ${ }^{17}$ on the accession of the evanescent light emanating from the guided mode in the core of a polished fiber half-block. With the use of an overlay whose optical 
properties can be modulated by an electrical signal, the device can be used for intensity modulation. Such devices utilizing liquid crystals ${ }^{17}$ for example, have been demonstrated to have low loss, high modulation depth, and moderate drive voltages. The phase and polarization modulation performance demonstrated here for the PVDF film with transparent ITO electrodes indicates the high potential application of PVDF to the all-fiber intensity modulator. Further, the birefringence properties of the PVDF film can be utilized for polarizing the guided light. If the birefringent indices are chosen such that one is higher and the other lower than the effective index of the fiber, the mode that sees the high index is not guided and the orthogonal mode seeing the low index is guided within the fiber. The fact that PVDF can be doped with the desired concentration of copolymers such as trifluoroethylene enhances its potential utility for such devices.

The same principle of accessing the evanescent light can also be utilized for the construction of a fiber-optic switch with the PVDF layer sandwiched in between two polished fiber half-blocks. The utility of the PVDF film in the transmission mode to twodimensional spatial phase modulation has been demonstrated. ${ }^{5}$ From the temporal performance of the PVDF film reported here, concurrent spatiotemporal modulation assumes significance for holographic interferometric systems that map the phase distribution over an extended surface. The IPM modulator can also be configured to function in a feedback mode to stabilize the phase or polarization in the presence of random drifts in optical sensors of the bulk-optic or fiber-optic kinds.

\section{Conclusion}

The PVDF-film-based phase and polarization modulator utilizing the optical properties of the film in the presence of an applied electric field has been characterized for the linearity of response at chosen frequencies, the frequency response, the phase-shifting coefficient, and the depth of polarization modulation. Through the use of the $J_{1} \ldots J_{4}$ method, a linear phase-shifting coefficient of $0.508 \mathrm{rad} / \mathrm{Vp}$ at $7.4 \mathrm{kHz}$ for the circularly clamped configuration and a polarization modulation depth of $0.326 \mathrm{rad} / \mathrm{Vp}$ at $8.4 \mathrm{kHz}$ for the freely supported configuration have been measured. The device performance of the different configurations of the IPM has demonstrated the generic utility of the transparent PVDF film for achieving a large dynamic range of phase and polarization modulation in a broad range of frequencies for use in optical sensor systems. Specifically, there exists a high potential for the utility of the PVDF film in all-fiber intensity modulators.

This research was partially supported by the Advanced Research Projects Agency through the Office of Naval Research. V. S. Sudarshanam thanks D. Port of Atochem Sensors for providing the piezofilm and J. Jackson of Metricon for the measurement of the indices and thickness of the piezofilm.

\section{References and Notes}

1. R. P. De Paula and E. L. Moore, "Review of all-fiber phase and polarization modulators," in Fiber Optic and Laser Sensors II, E. L. Moore and O. G. Ramer, eds., Proc. Soc. Photo-Opt. Instrum. Eng. 478, 3-11 (1984).

2. V. S. Sudarshanam and K. Srinivasan, "Linear readout of dynamic phase change in a fiber optic homodyne interferometer," Opt. Lett. 14, 140-142 (1989).

3. V. S. Sudarshanam and K. Srinivasan, "Phase shift nonlinearity at resonance in a piezofilm based fiber optic phase modulator," J. Appl. Phys. 68, 1975-1980 (1990).

4. V. S. Sudarshanam and R. O. Claus, "Frequency response and phase shift nonlinearity of a cylindrical PVDF film based fiber optic phase modulator," J. Lightwave Technol 11, 595-602 (1993).

5. T. Sato, Y. Ueda, and O. Ikeda, "Transmission type PVDF 2-D optical phase modulator,"Appl. Opt. 20, 343-350 (1981).

6. "Kynar piezofilm," Tech. Note (Atochem Sensors, Valley Forge, PA, 1990), p. 13.

7. V. S. Sudarshanam and R. O. Claus, "Split-cavity crosscoupled extrinsic fiber-optic interferometric sensor," Opt. Lett. 18, 543-545 (1993).

8. V. S. Sudarshanam, "Multimode fibre axial strain sensor utilizing end reflection interference," J. Mod. Opt. 39, 615-624 (1992).

9. V. S. Sudarshanam and S. V. Pappu, "Holographic optical element based single mode hybrid fiber optic interferometer for realizing zero order fringe," Fiber Integr. Opt. 11, 71-83 (1992).

10. R. M. A. Azzam and N. M. Bashara, Ellipsometry and Polarized Light (North-Holland, Amsterdam, 1977).

11. R. T. Denton, F. S. Chen, and A. A. Ballman, "Lithium tantalate light modulators," J. Appl. Phys. 38, 1611-1617 (1967).

12. D. K. Das-Gupta, K. Doughty, and D. B. Shier, "A study of structural and electrical properties of stretched polyvinylidene films," J. Electrostat. 7, 267-282 (1979).

13. J. H. McFee, J. G. Bergman, Jr., and G. R. Crane, "Pyroelectric and nonlinear optical properties of poled polyvinylidene fluoride films," Ferroelectrics 3, 305-313 (1972).

14. R. J. Shuford, A. F. Wilde, J. J. Ricca, and G. R. Thomas, "Characterization and piezoelectric activity of stretched and poled polyvinylidene fluoride. Part I: Effect of draw ratio and poling conditions," Polym. Eng. Sci. 16, 25-35 (1976).

15. "Model 2010 prism coupler,"Applications Note, Metricon Corp., Pennington, N.J., 1990.

16. H. Ogura and K. Kase, "Evaluation of the Lorentz factor of $\beta$-phase polyvinylidene fluoride crystals with the measured refractive index," Ferroelectrics 110, 145-156 (1990).

17. Z. K. Ioannidis, I. P. Giles, and C. Bowry, "All-fiber optic intensity modulators using liquid crystals," Appl. Opt. 30, 328-333 (1991).

18. J. C. Manifacier, J. Gasiot, and J. P. Fillard, "A simple method for the determination of the optical constants $n, k$ and the thickness of a weakly absorbing thin film," J. Phys. E. 9, 1002-1004 (1976).

19. W. A. Pliskin and E. E. Conrad, "Nondestructive determination of thickness and refractive index of transparent films," IBM J. Res. Dev. 8, 43-51 (1964).

20. K. Hane and S. Hattori, "Photothermal bending of a layered sample in plate form," Appl. Opt. 29, 145-150 (1990).

21. See, for example, R. O. B. Carpenter, "The electro-optic effect in uniaxial crystals of the dihydrogen phosphate type. III. Measurement of coefficients," J. Opt. Soc. Am. 40, 225-229 (1950).

22. D. Broussoux and F. Micheron, "Electro-optic and elasto-optic effects in polyvinylidene fluoride," J. Appl. Phys. 51, 20202023 (1980).

23. D. Gookin and R. Morris, "Electro-optic hysteresis in polyvinylidene fluoride,”Appl. Phys. Lett 45, 603-604 (1984). 


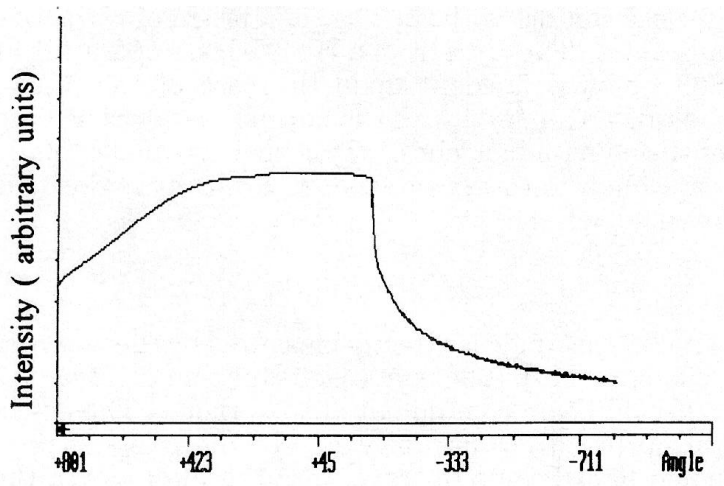




$$
d=\Delta N \lambda /\left[2 \mu\left(\cos r_{2}-\cos r_{1}\right)\right]
$$

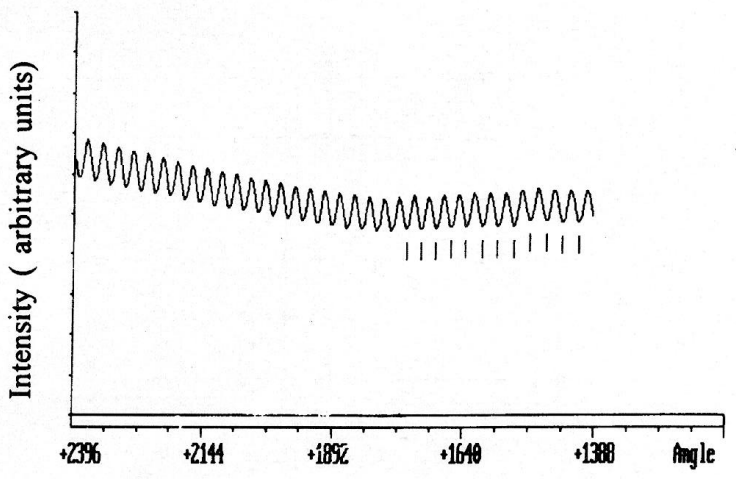

